Psychologie clinique

\title{
Comment les parents, les enseignants et les cliniciens évaluent les troubles du comportement externalisé du jeune enfant? Étude de la variabilité des jugements évaluatifs et de son impact sur le développement de l'enfant
}

\section{How do parents, teachers and clinicians assess young children's externalizing behaviour? Variation in their assessment and its impact on children's development}

\author{
I. Roskam ${ }^{\mathrm{a}, *}$, M. Stiévenart ${ }^{\mathrm{a}}$, J.-C. Meunier ${ }^{\mathrm{a}}$, \\ G. Van de Moortele ${ }^{a}$, P. Kinoo ${ }^{\text {b,c }}$, M.-C. Nassogne ${ }^{\text {b,d }}$ \\ a Institut des sciences psychologiques, faculté de psychologie, université catholique de Louvain, 10, place \\ Cardinal-Mercier, 1348 Louvain-la-Neuve, Belgique \\ ${ }^{\mathrm{b}}$ Cliniques universitaires Saint-Luc, 10, avenue Hippocrate, 1200 Bruxelles, Belgique \\ ${ }^{\mathrm{c}}$ Psychiatrie infantojuvénile, 10, avenue Hippocrate, 1200 Bruxelles, Belgique \\ d Neuropédiatrie, 10, avenue Hippocrate, 1200 Bruxelles, Belgique
}

Reçu le 30 avril 2009 ; accepté le 18 juin 2009

\section{Résumé}

Cette contribution questionne nos pratiques d'évaluation du comportement chez le jeune enfant. Les données concernant le comportement de 118 enfants ont été collectées par questionnaires et observation auprès de leurs parents, leurs enseignants et les cliniciens lors du recrutement et après 12 mois. Les résultats indiquent une variabilité importante entre les jugements évaluatifs des différents informateurs et un impact négatif de cette variabilité sur le développement ultérieur de l'enfant. Les résultats plaident en faveur d'une procédure multi-informateurs et multiméthodes dont les implications sont discutées sur le plan clinique. (C) 2009 Société française de psychologie. Publié par Elsevier Masson SAS. Tous droits réservés.

Mots clés : Troubles de comportement ; Évaluation ; Informateurs ; Méthodes

\footnotetext{
* Auteur correspondant.

Adresse e-mail : isabelle.roskam@uclouvain.be (I. Roskam).
} 


\begin{abstract}
Present study aims at refining assessment of preschoolers' behavior. Data was provided with questionnaires and observation on 118 preschoolers when recruited and 12 months later by their parents, their teachers and clinicians. The results confirmed high variations in informants' ratings as well as negative effects of such variations regarding later child's development. The results imply the need for a multi-informant and multimethod assessment of preschoolers' disruptive behavior. The implications of our findings for clinical purpose are discussed.
\end{abstract}

(C) 2009 Société française de psychologie. Published by Elsevier Masson SAS. All rights reserved.

Keywords: Disruptive behavior; Assessment; Disagreement; Informants; Methods

\title{
1. Introduction
}

Les difficultés de comportement dit externalisé (agitation, désobéissance, agressivité, opposition; voir Roskam et al., 2007 pour une description de ces difficultés) sont un motif de consultation récurrent auprès des services de santé mentale, des pédiatres, des neuropédiatres et des pédopsychiatres. Des auteurs rapportent que 25 à $40 \%$ des enfants en âge d'école maternelle présenteraient ce type de difficulté parmi lesquels 7 à $15 \%$ à des degrés modérés à sévères (Palfrey et al., 1985; Richman et al., 1982). L'évaluation de ce type de difficulté chez le jeune enfant constitue donc bien un objectif de première importance pour nos pratiques cliniques. En outre, des résultats d'études antérieures ont montré que ces difficultés tendent à persister dans l'enfance et l'adolescence avec des risques particuliers en matière de difficultés scolaires, d'insertion sociale auprès des pairs et de troubles dépressifs (Owens et Shaw, 2003 ; Richman et al., 1982). Dans ce contexte, cette contribution a pour objectif de questionner nos pratiques en matière d'évaluation du comportement chez le jeune enfant en mettant en évidence la variabilité existant entre les jugements évaluatifs des parents, des enseignants et des cliniciens ainsi qu'en questionnant l'impact possible de cette variabilité sur le développement ultérieur de l'enfant.

\subsection{La variabilité des jugements évaluatifs}

Tant dans les consultations que dans les études scientifiques, les parents sont souvent les informateurs privilégiés à propos du comportement de leur enfant. Ils passent en effet beaucoup de temps en sa présence et l'observent dans des situations et des contextes variés. Les parents sont également les informateurs les plus aisés à questionner : ils sont présents lors de la consultation clinique ou sont les premiers à être contactés par le chercheur. En outre, contrairement aux enfants d'âge scolaire ou aux adolescents, les jeunes enfants ne peuvent fournir une évaluation autorapportée de leur comportement.

Les études scientifiques rapportent pourtant des résultats forts et consistants concernant des différences systématiques entre les évaluations rapportées par les parents lors de l'évaluation du comportement de leur enfant et celles provenant d'autres informateurs. Lorsque les parents d'enfants tout-venant sont appelés à évaluer la personnalité de leur enfant, ils considèrent en effet leur enfant plus positivement que les enseignants (Roskam et al., 2001). En revanche, lorsque les parents sont amenés à évaluer les difficultés comportementales de leur enfant, que ce soit dans des échantillons tout-venant ou dans des échantillons cliniques, ils tendent à 
rapporter systématiquement plus de difficultés dans le comportement de l'enfant que l'enseignant (Achenbach et al., 1987; Grietens et al., 2004; Van der Ende et Verhulst, 2005; Winsler et Wallace, 2002). Ces différences systématiques conduisent à des corrélations modérées à élevées entre les deux parents mais faibles à modérées entre les parents et les enseignants (Achenbach et al., 1987 ; De los Reyes et Kazdin, 2005 ; Gross et al., 2004). À ce jour et à notre connaissance, aucune recherche n'a exploré la variabilité entre le jugement évaluatif des parents et celui des cliniciens ou entre celui des enseignants et celui des cliniciens.

\subsection{Comment expliquer la variabilité observée entre les informateurs?}

L'évaluation comportementale de l'enfant, a fortiori du jeune enfant, est extrêmement délicate. Plusieurs éléments liés aux caractéristiques de l'enfant lui-même, à celles de l'informateur, à celles de l'environnement auquel l'évaluation fait référence ou encore à l'interaction entre ces différents types de facteurs, peuvent être avancés pour rendre compte de cette variabilité.

De manière générale, il paraît évident que le contexte de référence dans lequel l'enfant est évalué joue un rôle essentiel. Le comportement de l'enfant évalué à partir de situations familiales par les parents pourrait ne pas correspondre en tout point à celui évalué à partir de situations scolaires par les enseignants ou encore à celui évalué lors d'une consultation clinique.

En outre, chaque informateur, professionnel ou non, évalue l'enfant en fonction de ce qu'il considère comme étant normal, attendu ou acceptable pour un enfant, fille ou garçon, à un âge donné. Les attentes ou les normes auxquelles les informateurs se réfèrent au cours de l'évaluation peuvent fortement varier. Là où les parents se référeront volontiers au comportement des autres enfants de la fratrie pour considérer que l'enfant-cible est plus agité, par exemple, que ne l'était l'aîné, les enseignants se réfèreront aux nombreux enfants qu'ils observent années après années dans leurs classes. Les cliniciens, quant à eux, se basent sur leurs connaissances théoriques du développement normal versus pathologique. L'influence du choix implicite d'un échantillon de référence sur l'évaluation produite par les différents informateurs est donc importante.

Ces attentes normatives peuvent également varier fortement d'un individu à un autre : chaque parent se montre plus ou moins tolérant à l'égard de l'agitation motrice ou de l'opposition du jeune enfant, par exemple, les enseignants et les cliniciens également. Au sein d'un couple, d'une école, voire même d'une équipe de cliniciens, la tolérance à l'égard du comportement externalisé du jeune enfant peut être sujette à controverse. Ces variations sont d'autant plus importantes que l'enfant est jeune. En effet, si les attentes à l'égard du comportement de l'enfant se précisent à mesure qu'il grandit, elles sont souvent floues à l'égard des plus jeunes. Qu'est-ce qu'une agitation normale ou qu'est-ce qu'une fréquence acceptable de gestes agressifs chez un petit de trois ans en sachant qu'une «certaine» agitation et une «certaine » agressivité sont normales à cette période de développement?

Ces attentes normatives, auxquelles se réfèrent les informateurs lorsqu'ils évaluent le comportement de l'enfant, peuvent également varier fortement d'une culture à une autre, en particulier, les normes liées aux rôles de genre. Ce qu'un groupe culturel jugera comme adéquat en matière d'assertivité ou d'agressivité physique peut en effet varier considérablement s'il s'agit d'une fille ou d'un garçon.

Un autre élément émotionnel et contextuel doit également être pris en considération pour rendre compte des variations entre les parents et d'autres informateurs. Les parents décidant de consulter auprès de cliniciens en raison de difficultés comportementales chez leur tout jeune enfant font une démarche extrêmement difficile : celle de reconnaître leur incapacité - temporaire ou permanente - à contenir les débordements de l'enfant et à lui imposer des limites. Une telle 
démarche fait souvent suite à d'autres tentatives pour solutionner les difficultés comportementales comme :

- modifier ses pratiques éducatives ;

- se documenter dans des ouvrages ou sur Internet;

- se faire conseiller par des personnes de confiance de l'entourage, etc.

Ces tentatives ont toutes échouées, en tout ou partiellement, ce qui amène les parents en consultation avec un ensemble d'attentes - souvent démesurées - à l'égard des traitements que pourrait proposer le clinicien. Dès lors, leur objectif est que le clinicien prenne les difficultés de l'enfant au sérieux et en mesure toute l'ampleur. C'est là que, lors de l'analyse de la demande, les parents vont sélectionner et présenter des épisodes mettant presque exclusivement l'accent sur ce qui dysfonctionne chez l'enfant. Le processus clinique lui-même amène les parents à se focaliser de manière quasi exclusive sur les difficultés de l'enfant. L'analyse de la demande, pratiquée lors de la première consultation, consiste bien à faire émerger les plaintes et motifs ayant conduit les parents à entamer la démarche d'aide. Il en va de même dans les recherches proposant aux parents des questionnaires portant exclusivement sur des symptômes psychopathologiques, tel que le très répandu Conners (Conners, 1982).

Enfin, des troubles émotionnels chez le parent ont également été associés à des effets négatifs systématiques sur l'évaluation du comportement de l'enfant. Plusieurs études ont à cet égard soutenu l'hypothèse de la «dépression-distorsion» impliquant que des mères déprimées surévalueraient les difficultés comportementales de leur enfant (Briggs-Gowan et al., 1996; Chi et Hinshaw, 2002; Grietens et al., 2004 ; Treutler et Epkins, 2003).

\subsection{Impact de la variabilité entre informateurs sur le développement de l'enfant}

Dans la mesure où elle reposerait sur des facteurs susceptibles d'influencer le pronostic d'évolution, la variabilité observée entre informateurs évaluant le comportement de l'enfant a récemment été présentée comme préjudiciable pour le développement ultérieur de ce dernier (Ferdinand et al., 2007). D'une part, si les parents rapportent davantage de difficultés à la maison que les enseignants à l'école, la cause de ces difficultés pourrait être attribuée à l'utilisation de pratiques éducatives inefficaces ou inconsistantes par les parents dans le cadre familial. D'autre part, si les parents rapportent moins de difficultés à la maison que les enseignants à l'école, il se pourrait que des difficultés spécifiques d'adaptation dans le cadre scolaire soient en cause et que ces difficultés reposent sur des compétences sociales et cognitives faibles chez l'enfant. Dans les deux cas, la variabilité entre informateurs serait un signe de risque compromettant le développement ultérieur de l'enfant. Il semble en tout cas qu'une évaluation consistante et une compréhension partagée des difficultés de l'enfant faciliteraient les efforts de collaboration entre parents, enseignants et cliniciens. À l'inverse, une évaluation trop contrastée et une compréhension discordante des difficultés de l'enfant conduiraient à l'échec de cette collaboration. Les résultats scientifiques dont nous disposons actuellement à propos de cette question sont peu nombreux et contradictoires (Ferdinand et al., 2004 ; Ferdinand et al., 2007).

\subsection{Les objectifs de notre étude}

L'étude rapportée ci-dessous mesure la concordance entre les jugements évaluatifs des parents, des enseignants et des cliniciens, tous évaluant le comportement d'enfants venus consulter en 
raison de troubles du comportement externalisé. Ensuite, l'impact de la variabilité observée entre les jugements évaluatifs des différents informateurs sur le développement ultérieur de l'enfant sera évalué.

\section{Méthode}

\section{1. Échantillon et procédure}

La présente contribution s'inscrit dans une vaste étude longitudinale portant sur les troubles externalisés du comportement chez le jeune enfant (voir le site http://www.uclouvain.be/h2mchildren.html pour une vision générale du programme de recherche).

Les données ont été récoltées dans un échantillon de 118 enfants, leurs parents, leurs enseignants et les cliniciens auprès desquels ils sont venus consulter. Tous les enfants ont été recrutés dans des services de pédiatrie ou de psychiatrie infantojuvénile, en particulier aux cliniques universitaires Saint-Luc à Bruxelles. Le motif exclusif de consultation consistait en des difficultés de comportement de type externalisé (agitation, opposition, agressivité, désobéissance). Les garçons sont au nombre de 92 et les filles, 26. L'âge des enfants varie entre 31 et 84 mois $(\mathrm{M}=50,89$; D.S. $=11,48)$. Au moment de la première consultation, tous les enfants fréquentaient l'enseignement ordinaire en communauté française de Belgique : 47 étaient en première maternelle, 39 en deuxième et 31 en troisième. La plupart des parents vivaient en couple $(75,1 \%)$, les autres vivaient séparément. Soixante-quinze pour cent des enfants avaient des frères et sœurs. Le nombre d'enfants dans la fratrie variait entre un et neuf $(25 \%$ d'enfants unique, $38 \%$ de fratries de deux enfants, $24 \%$ de fratries de trois enfants, $10 \%$ de fratries de quatre enfants et $3 \%$ de fratries comptant entre cinq et neuf enfants). Quarante-neuf pour cent des enfants étaient les aînés de leur fratrie, $30 \%$ les deuxièmes, $15 \%$ les troisièmes et $6 \%$ les quatrièmes ou au-delà.

Concernant le niveau de scolarisation des mères, $35 \%$ comptaient un maximum de 12 années, soit un niveau d'études secondaires, $49 \%$ avaient fait des études supérieures et $16 \%$ avaient un diplôme universitaire. Concernant le niveau de scolarisation des pères, $36 \%$ comptaient un maximum de 12 années, soit un niveau d'études secondaires, $42 \%$ avaient fait des études supérieures et $22 \%$ avaient un diplôme universitaire.

Trois assistants de recherche, tous licenciés en sciences psychologiques et comptant entre une et dix années d'expérience, ont été impliqués dans la récolte des données et entraînés à l'utilisation standardisée de la procédure de récolte des données. Au moment de la première consultation (temps 1 [T1]) et moyennant l'accord des parents, les enfants ont été évalués par un clinicien dans un local isolé. Les parents ont été reçus en entretien par un clinicien dans un autre local et ils ont été amenés à compléter-ensemble lorsqu'ils consultaient en couple-des questionnaires. Tous les enfants ont par ailleurs reçu la visite d'un clinicien à l'école. À cette occasion, un entretien avec l'enseignant a été conduit et l'enseignant a également complété des questionnaires. Au T1, 109 mères (92\% de l'échantillon), 93 pères (79\% de l'échantillon) et 90 enseignants (76\% de l'échantillon) ont participé à la récolte des données. La même procédure a été suivie 12 mois plus tard (temps 2 [T2]) ( $n=87 ; 74 \%$ de l'échantillon de départ). Au T2, 79 mères (91\% de l'échantillon), 64 pères ( $73 \%$ de l'échantillon) et 73 enseignants ( $84 \%$ de l'échantillon) ont participé à la récolte des données.

\subsection{Mesure du comportement de l'enfant}

Le comportement de l'enfant a été mesuré au moyen de plusieurs types d'instruments complétés par les parents, les enseignants et les cliniciens. Leur choix répond à une volonté de notre part de 
baser l'évaluation des troubles de comportement externalisé chez les enfants sur des instruments portant, d'une part, sur des comportements variés et représentatifs des jeunes enfants dans des situations de la vie quotidienne et, d'autre part, sur des instruments portant exclusivement sur des symptômes psychopathologiques associés aux troubles externalisés. Le choix de ces instruments répond également à la nécessité de varier les modes d'évaluation en fonction des informateurs. En effet, les cliniciens n'étant pas à même d'évaluer le comportement de l'enfant dans leur vie quotidienne, la nécessité de recourir à des mises en situation et à des techniques d'observation s'est imposée.

Le premier questionnaire a été complété par les parents et les enseignants : le profil socioaffectif (PSA, Dumas et al., 1997). Il comporte 80 items auxquels l'informateur doit répondre en termes d'intensité et de fréquence sur une échelle à six points allant de «jamais » à « toujours » (exemples d'items : «Exprime du plaisir à accomplir des choses », «Rit facilement », « Les enfants viennent le chercher pour jouer »). Le PSA est une mesure de l'adaptation générale des enfants, un score élevé reflétant une bonne adaptation comportementale. La version française du PSA a été validée auprès de 800 enfants et a démontré de très bonnes qualités psychométriques (LaFreniere et al., 1992). Dans notre échantillon, les indices de consistance interne (Cronbach's alpha $[\alpha]$ ) s'élevaient à 0,88 pour les parents et à 0,84 pour les enseignants. Le choix du PSA s'est imposé en raison de ses qualités psychométriques, mais également en raison de sa pertinence pour les tout jeunes enfants. Le PSA se présente en effet sous la forme d'une check-list de comportements représentatifs des enfants âgés de deux à six ans. Ce type d'instrument est utilisé de manière très fréquente dans les études empiriques, le plus répandu étant le Child behavior check-list (CBCL) d'Achenbach (1991). Le CBCL ne s'adresse cependant qu'aux enfants âgés de plus de quatre ans et convient aux enfants jusqu'à l'âge de 16 ans ce qui le rend peu spécifique à une période de développement donnée.

Un deuxième questionnaire a été utilisé auprès des parents et des enseignants : le SNAPIV (Swanson et al., 1992). Ce questionnaire mesure la présence des symptômes liés au déficit d'attention, à l'hyperactivité et au trouble oppositionnel tels qu'ils sont listés dans le DSM-IV (APA, 1996). Il comporte 23 items auxquels l'informateur doit répondre en termes d'intensité et de fréquence sur une échelle à quatre points allant de «pas du tout» à «tout à fait» (exemples d'items : «Éprouve des difficultés à rester attentif dans ses tâches ou ses jeux », «Remue sans cesse les mains ou les pieds ou se tortille sur son siège », «Éprouve des difficultés à attendre son tour »). Le SNAP-IV est une mesure des troubles du comportement chez l'enfant, un score élevé reflétant la présence d'un syndrome. Il démontre des qualités psychométriques satisfaisantes. Dans notre échantillon, les indices de consistance interne (Cronbach's alpha $[\alpha]$ ) s'élevaient à 0,62 pour les parents et à 0,72 pour les enseignants. Le choix du SNAP-IV s'est imposé en raison de son utilisation fréquente dans les consultations cliniques en raison de sa grande proximité avec le célèbre Conners (1982) mais en étant plus récent.

Le comportement de l'enfant a enfin été évalué par le clinicien au moyen d'un paradigme d'observation: le SNAP game (Hughes et al., 2002). La version originale de ce paradigme (Murray et al., 2001) a été mise au point dans le cadre de travaux portant sur l'impuissance apprise chez des enfants de mères dépressives. Il a ensuite été utilisé dans des travaux portant sur les troubles du comportement chez l'enfant, apparaissant dans ce contexte comme une procédure d'évaluation valide et efficace auprès de jeunes enfants (Hughes et al., 2002). Lors de la visite dans le cadre scolaire, l'enfant-cible était invité à choisir un enfant de sa classe avec lequel il souhaitait jouer. Les deux enfants étaient alors placés face au clinicien pour participer à ce jeu de cartes compétitif. La particularité de ce paradigme est que l'ordre des cartes proposées aux enfants est truqué de sorte que chacun des enfants à son tour, est placé dans une 
situation de frustration. Le jeu est enregistré sur vidéo permettant le codage, par le clinicien, des comportements de l'enfant-cible pendant les 19 tours de cartes que comporte le jeu. Chacun des tours de cartes a été codé séparément par deux codeurs entraînés et indépendants. L'accord entre les deux codeurs était élevé ; $r=0,94$ à 0,97 . Une variable relative au comportement adaptatif de l'enfant a été compilée à partir des interactions sociales et des affects positifs ; une autre variable relative aux aspects symptomatiques du comportement externalisé a été compilée à partir des affects négatifs, de l'agitation et de l'agressivité manifestés par l'enfant. Le choix de ce paradigme d'observation repose sur une revue de la littérature indiquant la rareté de ce type d'outil validé dans des études empiriques. En outre, le SNAP game présente un réel intérêt en termes d'applicabilité au contexte des consultations cliniques et de pertinence eu égard à notre population.

Toutes les variables issues du PSA, du SNAP-IV et du SNAP game ont été standardisées avec une moyenne de zéro et un écart-type de un. Des scores de différence standardisés ont ensuite été calculés pour évaluer les désaccords entre les différents informateurs selon la procédure de De los Reyes et Kazdin (2005). Ces scores de différence ont été calculés pour chacune des paires d'informateurs (parents-enseignants, parents-cliniciens, enseignants-cliniciens). Il s'agit de scores absolus indiquant la magnitude du désaccord entre deux informateurs. Un score de zéro indique dès lors un accord parfait entre les deux informateurs alors qu'un score élevé indique un désaccord élevé entre les deux informateurs.

\section{Résultats}

\subsection{Mesure de la concordance entre les jugements évaluatifs des parents, des enseignants et des cliniciens}

Des corrélations ont été calculées entre les évaluations faites par les parents, les enseignants et les cliniciens à propos du comportement de l'enfant. Des analyses séparées ont été réalisées selon que l'évaluation concerne des aspects adaptatifs du comportement de l'enfant (PSA + SNAP game ou interactions sociales et affects positifs pendant le jeu) et selon que l'évaluation concernant des aspects symptomatiques du comportement externalisé de l'enfant (SNAP-IV + SNAP game ou affects négatifs, agitation et agressivité pendant le jeu). Les résultats sont présentés dans le Tableau 1.

Des $t$-tests appliqués aux mesures répétées sur un même enfant par des évaluateurs indépendants, a également permis d'apprécier dans quelle mesure certains évaluateurs se distinguaient significativement des autres. Les résultats des $t$-tests sont présentés dans le Tableau 2.

Tableau 1

Corrélations entre les informateurs aux temps 1 et 2 (T1 et T2).

\begin{tabular}{|c|c|c|c|c|c|c|c|c|}
\hline & \multicolumn{4}{|c|}{$\begin{array}{l}\text { Aspects adaptatifs du } \\
\text { comportement }\end{array}$} & \multicolumn{4}{|c|}{$\begin{array}{l}\text { Aspects symptomatiques du } \\
\text { comportement externalisé }\end{array}$} \\
\hline & \multicolumn{2}{|c|}{ Parents } & \multicolumn{2}{|c|}{ Enseignants } & \multicolumn{2}{|c|}{ Parents } & \multicolumn{2}{|c|}{ Enseignants } \\
\hline & $\mathrm{T} 1$ & $\mathrm{~T} 2$ & $\mathrm{~T} 1$ & $\mathrm{~T} 2$ & $\mathrm{~T} 1$ & $\mathrm{~T} 2$ & $\mathrm{~T} 1$ & $\mathrm{~T} 2$ \\
\hline Parents & - & - & 0,18 & 0,16 & - & - & $-0,03$ & 0,16 \\
\hline Cliniciens & 0,02 & 0,03 & $-0,10$ & 0,20 & 0,06 & $-0,04$ & $0,22^{\mathrm{a}}$ & $0,34^{\mathrm{b}}$ \\
\hline
\end{tabular}

\footnotetext{
a $p<0,05$.

b $p<0,01$.
} 
Tableau 2

Résultats des $t$-tests (différence moyenne, déviation standard (D.S.), statistique ( $t$ ) comparant les évaluations au sein des différentes paires d'informateurs aux temps 1 et 2 (T1 et T2).

\begin{tabular}{|c|c|c|c|c|c|c|c|c|c|c|c|c|}
\hline & \multicolumn{6}{|c|}{ Aspects adaptatifs du comportement } & \multicolumn{6}{|c|}{ Aspects symptomatiques du comportement externalisé } \\
\hline & \multicolumn{3}{|l|}{$\mathrm{T} 1$} & \multicolumn{3}{|l|}{$\mathrm{T} 2$} & \multicolumn{3}{|l|}{$\mathrm{T} 1$} & \multicolumn{3}{|l|}{$\mathrm{T} 2$} \\
\hline & $\begin{array}{l}\text { Différence } \\
\text { moyenne }\end{array}$ & D.S. & $t$ & $\begin{array}{l}\text { Différence } \\
\text { moyenne }\end{array}$ & D.S. & $t$ & $\begin{array}{l}\text { Différence } \\
\text { moyenne }\end{array}$ & D.S. & $t$ & $\begin{array}{l}\text { Différence } \\
\text { moyenne }\end{array}$ & D.S. & $t$ \\
\hline Parents-enseignants & $-0,025$ & 1,29 & $-0,18$ & $-0,119$ & 1,24 & $-0,67$ & 0,011 & 1,39 & 0,08 & 0,259 & 1,21 & 1,62 \\
\hline Parents-cliniciens & 0,214 & 1,36 & 1,57 & $-0,287$ & 1,37 & $-1,62$ & $-1,218$ & 1,16 & $-10,35^{\mathrm{a}}$ & $-1,049$ & 1,24 & $-6,44^{\mathrm{a}}$ \\
\hline Enseignants-cliniciens & 0,237 & 1,32 & 1,66 & $-0,070$ & 1,31 & $-0,37$ & 0,248 & 1,33 & 1,89 & $-0,973$ & 1,02 & $-7,42^{\mathrm{a}}$ \\
\hline
\end{tabular}

a $p<0,001$. 
Les corrélations obtenues entre les informateurs évaluant les aspects adaptatifs du comportement de l'enfant sont très basses et non significatives. Lorsque les informateurs évaluent les aspects symptomatiques du comportement externalisé de l'enfant, les corrélations sont également très basses et non significatives hormis entre les enseignants et les cliniciens. Les coefficients atteignent, dans ce cas, un niveau modéré aux deux temps de mesure. Cela s'explique probablement en raison, d'une part, par une perspective professionnelle commune et, d'autre part, par un degré de précision plus élevé obtenu par les cliniciens dans l'évaluation des aspects symptomatiques du comportement externalisé avec le SNAP game que dans celle des aspects adaptatifs.

Les comparaisons effectuées à partir des $t$-tests indiquent, par ailleurs, que les différences au sein des différentes paires d'informateurs aux T1 et T2 ne sont pas systématiques en ce qui concerne les aspects adaptatifs du comportement. Les différences au sein des paires d'évaluateurs ne sont pas significatives. En ce qui concerne les aspects symptomatiques du comportement externalisé, en revanche, trois comparaisons sur six sont significatives. Elles indiquent des différences systématiques entre les cliniciens et les parents aux T1 et T2, d'une part, et entre les cliniciens et les enseignants au T2, d'autre part. La différence de moyenne montre que ce sont les cliniciens qui, plaçant l'enfant dans une situation de frustration telle qu'induite par le SNAP game, surévaluent les aspects symptomatiques du comportement externalisé des enfants.

\subsection{Impact de la variabilité entre informateurs sur le développement de l'enfant}

Afin de déterminer l'impact de la variabilité entre informateurs sur le développement de l'enfant, les scores de différence standardisés - reflétant les désaccords entre les différents informateurs - mesurés au T1 ont été entrés comme prédicteurs dans une analyse de régression avec comme variable à expliquer, le comportement externalisé de l'enfant mesuré au T2, soit 12 mois plus tard. Cette variable représentant le comportement externalisé de l'enfant est une variable latente reposant sur les aspects symptomatiques du comportement externalisé de l'enfant mesurés à partir du SNAP-IV complété par les parents, du SNAP-IV complété par l'enseignant et des affects négatifs, de l'agitation et de l'agressivité codés par le clinicien pendant le SNAP game. L'utilisation de cette variable latente permet de combiner les évaluations des différents informateurs en évitant de considérer la variabilité entre elles comme des erreurs de mesure à éliminer. En ce sens, elle offre la possibilité d'aborder la question de l'impact de cette variabilité sur le développement ultérieur de l'enfant sans avoir à opter pour l'un ou l'autre informateur au T2. La variable latente représentant le comportement externalisé de l'enfant a été calculée en s'appuyant sur les travaux de Kraemer et al. (2003). La procédure proposée par ces auteurs consiste à effectuer une analyse factorielle en composantes principales avec trois facteurs, l'un représentant le «trait», l'autre le «contexte» et le dernier, la «perspective». Le facteur «trait» est celui expliquant le plus de variance et sur lequel les différents informateurs saturent fortement. Le «trait» représente le comportement de l'enfant tel que tous les informateurs ont contribué à l'évaluer mais débarrassé des éléments liés au «contexte» dans lequel l'enfant a été évalué (général dans les situations de la vie quotidienne versus spécifique dans le paradigme d'observation) et liés à la «perspective» de chacun des informateurs (perspective professionnelle des enseignants et des cliniciens versus perspective émotionnelle des parents). Ces aspects de «contexte» et de «perspective» sont représentés par les deux autres facteurs résultant de l'analyse. Cette procédure multi-informateurs multi-méthodes a été éprouvée et validée dans une autre contribution à partir des données de notre échantillon (Roskam et al., sous presse). L'analyse en composante principale a permis d'obtenir trois facteurs, le premier représentant le comportement externalisé de l'enfant («trait»), le second, la «perspective» avec laquelle le comportement externalisé a 
Tableau 3

Résultats de l'analyse de régression où les désaccords entre informateurs au temps 1 (T1) prédisent le comportement externalisé de l'enfant au temps 2 (T2) (12 mois plus tard).

\begin{tabular}{lrrr}
\hline & \multicolumn{3}{c}{ Comportement externalisé (T2) } \\
\cline { 2 - 4 } & \multicolumn{1}{c}{$t$} & \multicolumn{1}{c}{$p$} \\
\hline Désaccord entre parents et enseignants (T1) & 0,28 & 1,81 & $\mathrm{a}$ \\
Désaccord entre parents et cliniciens (T1) & 0,16 & 1,01 & $\mathrm{~b}$ \\
Désaccord entre enseignants et cliniciens (T1) & $-0,45$ & $-2,81$ & \\
$R^{2}(\%)$ & 15,4 & & \\
\hline
\end{tabular}

${ }^{\mathrm{a}} p<0,010$.

b $p<0,01$.

été observé (perspective professionnelle non parentale-enseignants et cliniciens versus parentale non professionnelle-parents) et le troisième, le «contexte» au sein duquel le comportement externalisé a été observé (contexte général-SNAP-IV versus contexte spécifique-SNAP game). Le facteur «trait » « comportement externalisé de l'enfant »-dont il est fait usage dans le modèle de régression-explique $48 \%$ de la variance totale. Les saturations factorielles obtenues par les différents informateurs sur la variable latente « comportement externalisé de l'enfant » sont toutes supérieures à 0,40 , avec, respectivement, 0,41 pour les parents, 0,83 pour les enseignants et 0,76 pour les cliniciens.

Les résultats de l'analyse de régression présentés dans le Tableau 3, montrent que la relation entre les variables est bien linéaire $(F[3 ; 34]=3,24 ; p<0,05)$. Le pourcentage de variance expliquée par le modèle est de $15,4 \%$ ( $R^{2}$ ajusté). Le désaccord entre les parents et le clinicien ne prédit pas le comportement externalisé de l'enfant 12 mois plus tard de manière significative. En revanche, le désaccord entre les parents et l'enseignant $(\beta=0,28 ; p<0,10)$, d'une part, et le désaccord entre l'enseignant et le clinicien $(\beta=-0,45 ; p<0,01)$, d'autre part, prédisent le comportement externalisé de l'enfant 12 mois plus tard.

Ainsi, l'analyse montre que les enfants dont les parents et l'enseignant repèrent des comportements différents au T1, tendent à avoir un niveau de comportement externalisé élevé 12 mois plus tard. Le désaccord entre les parents et l'enseignant pourrait à ce titre être considéré comme problématique dans la mesure où il reposerait sur une vision peu homogène des difficultés de l'enfant ou sur une surévaluation des difficultés par l'un ou l'autre informateur. Dans ce cas, le désaccord entre informateurs pourrait être associé à des facteurs familiaux (tels que des pratiques éducatives inconsistantes) ou scolaires (tels que des difficultés sociales ou cognitives) susceptibles d'influencer le pronostic d'évolution des troubles externalisés du comportement chez l'enfant. L'autre résultat concernant l'enseignant et le clinicien est également intéressant : il indique qu'un degré d' accord élevé entre ces deux professionnels, au sujet du comportement externalisé de l'enfant, est effectivement lié à un niveau de comportement externalisé élevé 12 mois plus tard.

\section{Discussion}

La présente contribution avait pour objectif de mettre en évidence la variabilité existant entre les jugements évaluatifs des parents, des enseignants et des cliniciens ainsi que de questionner l'impact possible de cette variabilité sur le développement ultérieur de l'enfant. Elle a, pour ce faire, contribué à augmenter les connaissances antérieures en considérant le travail du clinicien 
dans le processus d'évaluation et en envisageant l'impact possible du désaccord entre les différents informateurs sur le développement ultérieur de l'enfant dans un design longitudinal.

En accord avec les données de la littérature, la variabilité entre les jugements évaluatifs des différents informateurs s'est révélée importante hormis entre les enseignants et les cliniciens lorsqu'ils évaluent les aspects symptomatiques du comportement externalisé. Cette exception est probablement due à la perspective professionnelle commune des enseignants et des cliniciens. Cette variabilité importante entre les jugements évaluatifs des différents informateurs doit nous inciter à la plus grande prudence dans nos pratiques. Ainsi, au vu de ces résultats, il paraît inacceptable, aussi bien pour des objectifs cliniques que de recherche, de baser l'évaluation comportementale des enfants agités, agressifs, opposants ou désobéissants sur des questionnaires, tels que, par exemple, le PSA (Dumas et al., 1997), le SNAP-IV (Swanson et al., 1992) ou encore le Conners (Conners, 1982), proposés uniquement au parent présent lors de la consultation. L'évaluation comportementale d'un enfant est un processus complexe qui requiert du temps et une formation professionnelle spécifique. Ce processus requiert toujours une prise de contact avec l'enseignant de l'école fréquentée par l'enfant et interagissant avec lui au quotidien. Il requiert aussi l'utilisation de techniques d'observation par le clinicien lui permettant de se forger sa propre opinion sans qu'elle puisse être d'emblée orientée par le discours parental ou scolaire.

Aussi, la complexité du processus de l'évaluation comportementale doit dans les faits, refléter la complexité du comportement lui-même car l'enfant en relation avec son père ou avec sa mère, n'est ni exactement l'enfant en classe ou en cours de récréation, ni exactement celui que nous rencontrons en consultation. Le comportement de l'enfant doit être considéré comme une synthèse de toutes ces composantes.

Les résultats obtenus en ce qui concerne l'impact du désaccord entre informateurs sur le développement ultérieur de l'enfant suggèrent également quelques pistes de réflexion pour nos pratiques cliniques. Ces résultats apportent un autre élément en faveur d'une implication systématique des enseignants dans le processus d'évaluation. En effet, nos résultats indiquent que leur contribution telle que mise en perspective avec celle des parents et celle des cliniciens apporte une information indispensable. D'une part, le désaccord entre parents et enseignants nous renseigne sur une possible difficulté de considérer les difficultés de l'enfant sous le même angle. Cette variabilité dans les jugements évaluatifs est dans les faits, souvent la source d'un dialogue difficile à établir entre l'école et la famille. L'enseignant comme les parents peuvent se sentir incompris, voire peu respectés, dans la manière dont ils perçoivent le comportement de l'enfant. Cela peut dès lors avoir pour conséquence, une collaboration inefficace, pouvant se révéler préjudiciable pour l'éducation et le développement de l'enfant. À cet égard, les cliniciens ont peut-être un rôle crucial à jouer en considérant que leur travail permet un recueil systématique des évaluations de chacun avec une volonté unique de dégager des pistes d'aide pour l'enfant. Ils pourraient en ce sens être les facilitateurs d'une communication entre parents et enseignants visant non pas à décider quel informateur a tort ou a raison sur les comportements de l'enfant, mais à attirer l'attention de tous sur la complexité du comportement de cet enfant en développement dans ses milieux de vie. En outre, les résultats suggèrent la pertinence des évaluations fournies par les enseignants qui, lorsqu'elles sont en accord avec celles des cliniciens, convergent vers une prédiction des difficultés ultérieures de comportement chez l'enfant. Comme nous l'évoquions dans la partie introductive de cette contribution, il est probable que l'évaluation des comportements de l'enfant par les enseignants s'avère plus pertinente, ce que montre nos données, car ils disposent, dans leur cadre de travail, d'un échantillon de comparaison composé de plusieurs dizaines ou centaines d'enfants ainsi que d'une distance émotionnelle plus grande que celle des parents par rapport à l'enfant-cible. Cela fait des enseignants des partenaires professionnels privilégiés 
pour les cliniciens amenés à conduire une évaluation précoce du comportement externalisé d'un enfant.

Enfin, si la présente contribution a bien permis de questionner nos pratiques en matière d'évaluation du comportement chez le jeune enfant, d'autres recherches sont encore nécessaires. Elles auraient pour but de répliquer les résultats obtenus avec nos données empiriques dans des échantillons d'enfants présentant d'autres types de symptômes ainsi qu'à d'autres périodes de développement. En effet, à l'heure actuelle, peu de choses sont connues sur les questions liées à la variabilité entre informateurs évaluant d'autres types de troubles. Par exemple, la question reste entière concernant la spécificité de l'évaluation des troubles du comportement dit externalisé par rapport à celle des troubles dits internalisés ou encore à celle de syndromes spécifiques. De la même façon, les questions liées à la variabilité entre informateurs à d'autres périodes de développement restent d'actualité, comme par exemple, entre l'évaluation précoce des troubles de comportement par rapport à celle menée auprès d'enfants d'âge scolaire (six à 12 ans) ou bien d'adolescents.

\section{Conflits d'intérêts}

\section{Aucun.}

\section{Références}

American Psychiatric Association, 1996. DSM IV : Manuel statistique et diagnostique des troubles mentaux. Masson, Paris.

Achenbach, T.M., 1991. Manual for the CBCL/4-18 and 1991 Profile. University of Vermont, Department of Psychiatry, Burlington.

Achenbach, T.M., McConaughy, S.H., Howell, C.T., 1987. Child/adolescent behavioral and emotional problems: implications of cross-informant correlations for situational specificity. Psychological bulletin 101, 213-232.

Briggs-Gowan, M.J., Carter, A.S., Schwab-Stone, M., 1996. Discrepancies among mother, child, and teacher reports: examining the contributions of maternal depression and anxiety. Journal of abnormal child psychology 24 (6), $749-765$.

Chi, T.C., Hinshaw, S.P., 2002. Mother-child relationships of children with ADHD: the role of maternal depressive symptoms and depression-related distortions. Journal of abnormal child psychology 30 (4), 387-400.

Conners, C.K., 1982. Parent and teacher rating forms for the assessment of hyperkineses in children. In: Keller, P.A., Ritt, L.G. (Eds.), Innovation in clinical practice. FI Professionnel Research Exchange Inc, Sarrasota.

De los Reyes, A., Kazdin, A.E., 2005. Informant discrepancies in the assessment of childhood psychopathology: a critical review, theoretical framework, and recommendations for further study. Psychological bulletin 131 (4), $483-509$.

Dumas, J., LaFrenière, P., Capuano, F., Durning, P., 1997. Profil socio-affectif (PSA). Évaluation des compétences sociales et des difficultés d'adaptation des enfants de $21 / 2$ à 6 ans. Éditions du centre de psychologie appliquée, Paris.

Ferdinand, R.F., van der Ende, J., Verhulst, F.C., 2007. Parent-teacher disagreement regarding behavioral and emotional problems in referred children is not a risk factor for poor outcome. European child and adolescent psychiatry 16, 121-127.

Ferdinand, R.F., van der Ende, J., Verhulst, F.C., 2004. Parent-adolescent disagreement regarding psychopathology in adolescents from the general population as a risk factor for adverse outcome. Journal of abnormal psychology 113 , 198-206.

Grietens, H., Onghena, P., Prinzie, P., Gadeyne, E., Van Assche, V., Ghesquière, P., et al., 2004. Comparison of mothers', fathers', and teachers' reports on problem behavior in 5- to 6-year-old children. Journal of psychopathology and behavioral assessment 26 (2), 137-146.

Gross, D., Fogg, L., Garvey, C., Julion, W., 2004. Behavior problems in young children: an analysis of cross-informant agreements and disagreements. Research in nursing and health 27, 413-425.

Hughes, C., Oksanen, H., Taylor, A., Jackson, J., Murray, L., Caspi, A., et al., 2002. I'm gonna beat you!" SNAP!: an observational paradigm for assessing young children's disruptive behavior in competitive play. Journal of child psychology and psychiatry 43 (4), 507-516. 
Kraemer, H.C., Measelle, J.R., Ablow, J.C., Essex, M.J., Boyce, W.T., Kupfer, D.J., 2003. A new approach to integrating data from multiple informants in psychiatric assessment and research: mixing and matching contexts and perspectives. American journal of psychiatry 160 (9), 1566-1577.

LaFreniere, P.J., Dumas, J.E., Capuano, F., Dubeau, D., 1992. Development and validation of the preschool socioaffective profile. Psychological assessment 4 (4), 442-450.

Murray, L., Woolgar, M., Cooper, P., Hipwell, A., 2001. Cognitive vulnerability to depression in five year old children of depressed mothers. Journal of child psychology and psychiatry 42, 891-900.

Owens, E.B., Shaw, D.S., 2003. Predicting growth curves of externalizing behavior across the preschool years. Journal of abnormal child psychology 31 (6), 575-590.

Palfrey, J.S., Levine, M.D., Walker, D.K., Sullivan, M., 1985. The emergence of attention deficit in early childhood: a prospective study. Journal of developmental and behavioral pediatrics 6, 339-348.

Richman, N., Stevenson, J., Graham, P., 1982. Pre-school to school: A behavioral study. Academic Press, London.

Roskam, I., Kinoo, P., Nassogne, M.-C., 2007. L'enfant avec troubles externalisés du comportement : approche épigénétique et développementale. Neuropsychiatrie de l'enfance et de l'adolescence 55, 204-214.

Roskam, I., Meunier, J.-C., Stiévenart, M., Van de Moortele, G., Kinoo, P., Nassogne, M.-C. (sous presse). Le diagnostic précoce des troubles du comportement externalisé est-il fiable? Mise à l'épreuve d'une procédure multi informateurs et multi méthodes.

Roskam, I., Vandenplas-Holper, C., de Maere-Gaudissart, A., 2001. Mothers' and teachers' ratings of the child's personality. Child's age, gender, scholastic achievement, mother's educational level, and rater effects. European review of applied psychology 51 (4), 289-303.

Swanson, J.M., Nolan, E.E., Pelham, W.E., 1992. SNAP-IV. University of California (http://www.adhd.net/ [consulté le 2 octobre 2005]).

Treutler, C.M., Epkins, C.C., 2003. Are discrepancies among child, mother, and father reports on children's behavior related to parents' psychological symptoms and aspects of parent-child relationships? Journal of abnormal child psychology 31 (1), 13-27.

Van der Ende, Verhulst, F.C., 2005. Informant, gender and age differences in rating of adolescent problem behavior. European child and adolescent psychiatry 14, 117-126.

Winsler, A., Wallace, G.L., 2002. Behavior problems and social skills in preschool children: parent-teacher agreement and relations with classroom observations. Early education and development 13 (1), 41-58. 